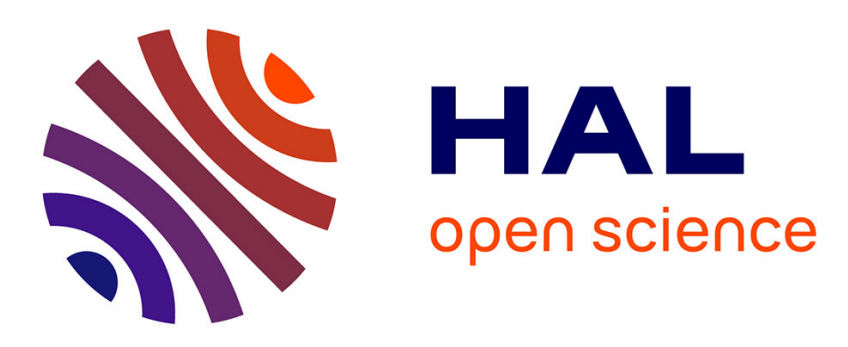

\title{
On Maximal Chain Subgraphs and Covers of Bipartite Graphs
}

\author{
Tiziana Calamoneri, Mattia Gastaldello, Arnaud Mary, Marie-France Sagot, \\ Blerina Sinaimeri
}

\section{- To cite this version:}

Tiziana Calamoneri, Mattia Gastaldello, Arnaud Mary, Marie-France Sagot, Blerina Sinaimeri. On Maximal Chain Subgraphs and Covers of Bipartite Graphs. Combinatorial Algorithms - 27th International Workshop, IWOCA 2016, Aug 2016, Helsinki, Finland. pp.137-150, 10.1007/978-3-319-445434_11. hal-01388546

\section{HAL Id: hal-01388546 \\ https://hal.inria.fr/hal-01388546}

Submitted on 30 May 2017

HAL is a multi-disciplinary open access archive for the deposit and dissemination of scientific research documents, whether they are published or not. The documents may come from teaching and research institutions in France or abroad, or from public or private research centers.
L'archive ouverte pluridisciplinaire HAL, est destinée au dépôt et à la diffusion de documents scientifiques de niveau recherche, publiés ou non, émanant des établissements d'enseignement et de recherche français ou étrangers, des laboratoires publics ou privés. 


\title{
On Maximal Chain Subgraphs and Covers of Bipartite Graphs
}

\author{
Tiziana Calamoneri ${ }^{1}$, Mattia Gastaldello ${ }^{1,2}$, Arnaud Mary ${ }^{2}$, Marie-France Sagot ${ }^{2}$, and \\ Blerina Sinaimeri ${ }^{2}$ \\ 1 Sapienza University of Rome \\ via Salaria 113, 00198 Roma, Italy. \\ 2 INRIA and Université de Lyon \\ Université Lyon 1, LBBE, CNRS UMR558, France.
}

\begin{abstract}
In this paper, we address three related problems. One is the enumeration of all the maximal not necessarily induced chain subgraphs of a bipartite graph, for which we provide a polynomial delay algorithm. We give bounds on the number of maximal chain subgraphs for a bipartite graph and use them to establish the input-sensitive complexity of the enumeration problem. The second problem we treat is the one of finding the minimum number of chain subgraphs needed to cover all the edges a bipartite graph. For this we provide an exact exponential algorithm with a non trivial complexity. Finally, we approach the problem of enumerating all minimal chain subgraph covers of a bipartite graph and show that it can be solved in quasi-polynomial time.

Keywords: Chain Subgraph Cover Problem, Enumeration Algorithms, Exact exponential algorithms.
\end{abstract}

\section{Introduction}

Enumerating (listing) the subgraphs of a given graph plays an important role in analysing its structural properties. It thus is a central issue in many areas, notably in data mining and computational biology.

In this paper, we address the problem of enumerating without repetitions all maximal not necessarily induced chain subgraphs of a bipartite graph. These are graphs that do not contain a $2 K_{2}$ as induced subgraph. From now on, we will refer to them as chain subgraphs for short when there is no ambiguity.

Bipartite graphs arise naturally in many applications, such as biology as will be mentioned later in the introduction, since they enable to model the relations between two different classes of objects. The problem of enumerating in bipartite graphs all subgraphs with certain properties has thus already been considered in the literature. These concern for instance maximal bicliques for which polynomial delay enumeration algorithms in bipartite $[6,11]$ as well as in general graphs $[5,11]$ were provided. In the case of maximal induced chain subgraphs, their enumeration can be done in output polynomial time as it can be reduced to the enumeration of a particular case of the minimal hitting set problem [7] (where the sets in the family are of cardinality 4). However, the existence of a polynomial delay algorithm for this problem remains open. To the best 
of our knowledge, nothing is known so far about the problem of enumerating maximal not necessarily induced chain subgraphs in bipartite graphs.

In this paper, we propose a polynomial delay algorithm to enumerate all maximal chain subgraphs of a bipartite graph. We also provide an analysis of the time complexity of this algorithm in terms of input size. In order to do this, we prove some upper bounds on the maximum number of maximal chain subgraphs of a bipartite graph $G$ with $n$ nodes and $m$ edges. This is also of intrinsic interest as combinatorial bounds on the maximum number of specific subgraphs in a graph are difficult to obtain and have received a lot of attention (see for e.g. [8, 12]).

We then address a second related problem called the minimum chain subgraph cover problem. This asks to determine, for a given graph $G$, the minimum number of chain subgraphs that cover all the edges of $G$. This has already been investigated in the literature as it is related to other well-known problems such as maximum induced matching (see e.g. [3, 4]). For bipartite graphs, the problem was shown to be NP-hard [14].

Calling $m$ the number of edges in the graph, we provide an exact exponential algorithm which runs in time $O^{*}\left((2+\varepsilon)^{m}\right)$, for every $\varepsilon>0$ by combining our results on the enumeration of maximal chain subgraphs with the inclusion-exclusion technique [1] (by $O^{*}$ we denote standard big $O$ notation but omitting polynomial factors). Notice that, since a chain subgraph cover is a family of subsets of edges, the existence of an algorithm whose complexity is close to $2^{m}$ is not obvious. Indeed, the basic search space would have size $2^{2^{m}}$, which corresponds to all families of subsets of edges of a graph on $m$ edges.

Finally, we approach the problem of enumerating all minimal covers by chain subgraphs. To this purpose, we provide a quasi-polynomial time algorithm to enumerate all minimal covers by maximal chain subgraphs of a bipartite graph. To do so, we prove that this can be polynomially reduced to the enumeration of the minimal set covers of a hypergraph.

Besides their theoretical interest, the problems of finding one minimum chain subgraph cover and of enumerating all such covers have also a direct application in biology. Nor et al. [13] showed that a minimum chain subgraph cover of such a bipartite graph provides a good model for identifying the minimum genetic architecture enabling to explain one type of manipulation, called cytoplasmic incompatibility, by bacteria of a genus called Wolbachia of their insect hosts. This phenomenon, results in the death of embryos produced in crosses between males carrying the infection and uninfected females. The observed cytoplasmic compatibility relationships, can be then represented by a bipartite graph with males and females in different classes. Moreover, as different minimum covers may correspond to solutions that differ in terms of their biological interpretation, the capacity to enumerate all such minimum chain covers becomes crucial.

The remainder of the paper is organised as follows. In Section 2, we give some definitions and preliminary results that will be used throughout the paper. Section 3 then provides a polynomial delay algorithm to enumerate all maximal chain subgraphs in a bipartite graph $G$ with $n$ nodes and $m$ edges, and Section 4 presents an upper bound on their maximum number. We use the latter to further establish the input-sensitive complexity of the enumeration algorithm. In Section 5, we detail the exact algorithm for finding a minimum chain cover in bipartite graphs, and in Section 6 we exploit the 
connection of this problem with the minimal set cover of a hypergraph to show that it is possible to enumerate in quasi-polynomial time all minimal covers by maximal chain subgraphs of a bipartite graph. Finally, we conclude with some open problems.

\section{Preliminaries}

Throughout the paper, we assume that the reader is familiar with the standard graph terminology, as contained for instance in [2]. We consider finite undirected graphs without loops or multiple edges. For each of the graph problems in this paper, we let $n$ denote the number of nodes and $m$ the number of edges of the input graph.

Given a bipartite graph $G=(U \cup W, E)$ and a node $u \in U$, we denote by $N_{G}(u)$ the set of nodes adjacent to $u$ in $G$ and by $E_{G}(u)$ the set of edges incident to $u$ in $G$. Moreover, given $U^{\prime} \subseteq U$ and $W^{\prime} \subseteq W$, we denote by $G\left[U^{\prime}, W^{\prime}\right]$ the subgraph of $G$ induced by $U^{\prime} \cup W^{\prime}$. A node $u \in U$ such that $N_{G}(u)=W$ is called a universal node.

A bipartite graph is a chain graph if it does not contain a $2 K_{2}$ as an induced subgraph. Equivalently, a bipartite graph is a chain graph if and only if, for each two nodes $v_{1}$ and $v_{2}$ both in $U$ (resp. in $W$ ), it holds that either $N_{G}\left(v_{1}\right) \subseteq N_{G}\left(v_{2}\right)$ or $N_{G}\left(v_{2}\right) \subseteq$ $N_{G}\left(v_{1}\right)$. Given a chain subgraph $C=(X \cup Y, F)$ of $G$, we say that a permutation $\pi$ of the nodes of $X$ is a neighbourhood ordering of $C$ if $N_{C}\left(u_{\pi(1)}\right) \subseteq N_{C}\left(u_{\pi(2)}\right) \subseteq \ldots \subseteq$ $N_{C}\left(u_{\pi(|U|)}\right)$. A permutation is just an ordered arrangement of a set of nodes. Observe that if $X \subset U$, the sets $N_{C}\left(u_{\pi(1)}\right), \ldots, N_{C}\left(u_{\pi(l)}\right)$ for some integer $l \leq|U|$, may be empty. By the largest neighbourhood of $C$, we mean the neighbourhood of a node $x$ in $X$ for which the set $N_{C}(x) \subseteq Y$ has maximum cardinality. A set $Y^{\prime} \subseteq Y$ is a maximal neighborhood of $G$, if there exists $x \in X$ such that $N_{G}(x)=Y^{\prime}$ and there does not exist a node $x^{\prime} \in X$ such that $N_{G}(x) \subset N_{G}\left(x^{\prime}\right)$. Two nodes $x, x^{\prime}$ such that $N_{C}(x)=N_{C}\left(x^{\prime}\right)$ are called twins.

In this paper, we always consider non induced chain subgraphs of a graph $G$. Hence, we identify a chain subgraph $C$ of $G$ by its set of edges $E(C) \subseteq E(G)$ and in that case its set of nodes will be constituted by all the nodes of $G$ incident to at least one edge in $C$ (sometimes abusing notation, we more simply write $C \subseteq G$ or $e \in C$ ). A maximal chain subgraph $C$ of a given bipartite graph $G$ is a connected chain subgraph such that no superset of $E(C)$ is a chain subgraph. We denote by $C(G)$ the set of all maximal chain subgraphs in $G$.

A set of chain subgraphs $C_{1}, \ldots, C_{k}$ is a cover for $G$ if $\cup_{1 \leq i \leq k} E\left(C_{i}\right)=E(G)$. Observe that, given any cover of $G$ by chain subgraphs $C=\left\{C_{1}, \ldots C_{k}\right\}$, there exists another cover of same size $C^{\prime}=\left\{C_{1}^{\prime}, \ldots C_{k}^{\prime}\right\}$ whose chain subgraphs are all maximal; more precisely, for each $i=1, \ldots, k, C_{i}^{\prime}$ is a maximal chain subgraph of $G$ and $C_{i}^{\prime}$ admits $C_{i}$ as subgraph. In order to avoid redundancies, from now on, although not explicitly highlighted, we will restrict our attention to the covers by maximal chain subgraphs.

We denote by $\mathcal{S}(G)$ the set of all minimal chain covers of a bipartite graph $G$.

An enumeration algorithm is said to be output polynomial or total polynomial if the total running time is polynomial in the size of the input and the output. It is said to be polynomial delay if the time between the output of any one solution and the next one is bounded by a polynomial function of the input size [10]. 


\section{Enumerating All Maximal Chain Subgraphs}

In this section, we provide a polynomial delay algorithm for enumerating all the maximal chain subgraphs of a given bipartite graph. We start by proving the following result.

Proposition 1. Let $C=(X \cup Y, F)$ be a chain subgraph of $G=(U \cup W, E)$, with $X \subseteq U$, $Y \subseteq W$ and $F \subseteq E$, and let $x \in X$ be a node with largest neighbourhood in $C$. Then $C$ is a maximal chain subgraph of $G$ if and only if:

(i) $N_{C}(x)=N_{G}(x)$ is a maximal neighbourhood of $G$, i.e. there does not exist a node $x^{\prime} \in X$ such that $N_{G}(x) \subset N_{G}\left(x^{\prime}\right)$.

(ii) $C \backslash E_{G}(x)$ is a maximal chain subgraph of $G\left[U \backslash\{x\}, N_{G}(x)\right]$.

Proof. $(\Rightarrow)$ Let $C=(X \cup Y, F)$ be a maximal chain subgraph of $G=(U \cup W, E)$. To prove that $(i)$ holds, suppose by contradiction that $N_{C}(x)$ is not a maximal neighbourhood of $G$, i.e. there exists $x^{\prime} \in U$ with $N_{C}(x) \subset N_{G}\left(x^{\prime}\right)$ (possibly $x^{\prime}=x$ ). Since $N_{C}(x)$ is the largest neighbourhood of $C$, for all $z \in X$, we have $N_{C}(z) \subseteq N_{C}(x) \subset N_{G}\left(x^{\prime}\right)$, so we can then add to $C$ all the edges incident to $x^{\prime}$ and still obtain a chain subgraph thereby contradicting the maximality of $C$. To prove that (ii) holds, first observe that $N_{G}(x)=Y$ (otherwise we would violate (i) with $x^{\prime}=x$ ). By contradiction, assume that $C \backslash E_{G}(x)$ is not maximal in $G\left[U \backslash\{x\}, N_{G}(x)\right]$. Then, there exists a chain subgraph $C^{\prime}$ such that $C \backslash E_{G}(x) \subset C^{\prime} \subseteq G\left[U \backslash\{x\}, N_{G}(x)\right]$. By adding to each one of the previous graphs the edges in $E_{G}(x)$, we have that the strict inclusion is preserved because the added edges were not present in any one of the three graphs. Since $C^{\prime}$ with the addition of $E_{G}(x)$ is still a chain subgraph with $N_{G}(x)$ as its largest neighbourhood, we reach a contradiction with the hypothesis that $C$ is maximal in $G$.

$(\Leftarrow)$ We show that if both $(i)$ and $(i i)$ hold, then the chain subgraph $C$ of $G$ is maximal. Suppose by contradiction that $C$ is not maximal in $G$, and let $C^{\prime}$ be a chain subgraph of $G$ such that $C \subset C^{\prime}$. Let $x$ be the node with the largest neighbourhood in $C$. It follows that $N_{C}(x) \subseteq N_{C^{\prime}}(x)$. As $(i)$ holds, we have that $N_{G}(x)=N_{C}(x) \subseteq N_{C^{\prime}}(x) \subseteq N_{G}(x)$ from which we derive that $N_{C^{\prime}}(x)=N_{G}(x)$, and that $C^{\prime} \subseteq G\left[U, N_{G}(x)\right]$ since $N_{C^{\prime}}(x)$ is a maximal neighbourhood of $G$, hence the largest neighbourhood of $C^{\prime}$ (and $C$ by the hypothesis). This implies also that $C$ and $C^{\prime}$ differ in some node different from $x$, i.e. $C \backslash E_{G}(x) \subset C^{\prime} \backslash E_{G}(x) \subseteq G\left[U \backslash\{x\}, N_{G}(x)\right]$. Notice that $C^{\prime} \backslash E_{G}(x)$ is still a chain subgraph because we simply removed node $x$ and all its incident edges. We then get a contradiction with (ii).

Proposition 1 leads us to the design of Algorithm 1 which efficiently enumerates all maximal chain subgraphs of $G$. It exploits the fact that, in each maximal chain subgraph, a node $u$ whose neighbourhood is largest is also maximal in $G$ (part (i) of Proposition 1) and this holds recursively in the chain subgraph obtained by removing node $u$ and restricting the graph to $N_{C}(u)$ (part (ii) of Proposition 1). To compute the maximal neighbourhood nodes, the algorithm uses a function, computeCandidates, that, given sets $U$ and $W$, returns for each maximal neighbourhood $Y \subset W$, a unique node $u$, called candidate, for which $N_{G}(u)=Y$. This means that in case of twin nodes, the function computeCandidates extracts only one representative node according to some fixed order on the nodes (e.g. the node with the smallest label according to the lexicographical order). If the graph has no edges, the function returns the empty set. 
Proposition 2 (Correctness). Algorithm 1 correctly enumerates all the maximal chain subgraphs of the input graph $G$ without repetitions.

Proof. Let $G=(U \cup W, E)$ be a bipartite graph. We prove the correctness of Algorithm 1 by induction on $|U|$, i.e. we show that all the solutions are output, without repetitions.

When $|U|=1$, let $u$ be the only node in $U$. We have that $N_{G}(u)$ is the only neighbourhood in $W$, and line 3 returns $\{u\}$ as unique candidate. In line 9, the algorithm reduces the graph of interest. In line 10 , the whole $E_{G}(u)$ is added to the current chain subgraph $C$. Then the function is recursively recalled, with $U^{\prime}=\emptyset$ so the condition at line 4 is true and $C$ is printed; it is in fact the only chain subgraph of $G$, and it is trivially maximal. No repetitions hold. Correctness then follows when $|U|=1$.

Assume now that $|U|=k$ with $k>1$. As inductive hypothesis, let the algorithm work correctly when $|U|=k-1$.

For each candidate $u$, the algorithm recursively recalls the same function on a reduced graph and, by the inductive hypothesis, outputs all chain subgraphs of this reduced subgraph without repetitions. By Proposition 1, if we add to each one of these chain subgraphs the node $u$ and all the edges incident to $u$ in $G[U, W]$, we get a different maximal chain subgraph of $G$ since each maximal chain subgraph has one and only one maximal neighborhood and the function computeCandidates returns only one representative node. Recall that in the case of twin nodes the algorithm will always consider the nodes in a precise order and so no repetition occurs. Moreover, iterating this process for all candidates guarantees that all maximal chain subgraphs are enumerated and no one is missed.

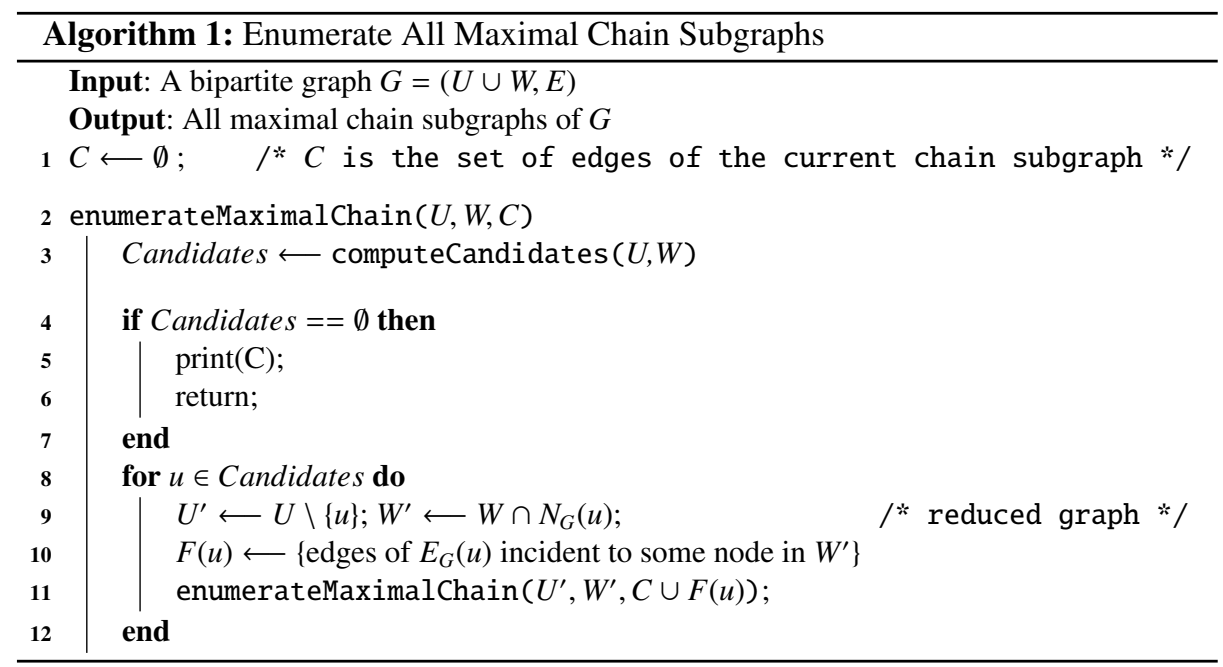

Let $G=(U \cup W, E)$ be a bipartite graph, with $n=|U|+|W|$ and $m=|E|$. Before proving the time complexity of Algorithm 1, we observe that the running time of the function ComputeCandidates is $O(\mathrm{~nm})$. Indeed, if we assume that the adjacency lists of the graph are ordered, for each node $u_{i} \in U$, it requires only time proportional to $i \cdot \operatorname{deg}\left(u_{i}\right) \leq n \cdot \operatorname{deg}\left(u_{i}\right)$ to check whether the neighbourhood of $u_{i}$ either is included, or includes the neighbourhood of $u_{j}$, for each $j<i$. 
Proposition 3 (Time Complexity and Polynomial Delay). Let $G=(U \cup W, E)$ be a bipartite graph. The total running time of Algorithm 1 is $O\left(|C(G)| n^{2} m\right)$. Moreover, the solutions are enumerated in polynomial time delay $O\left(n^{2} m\right)$.

Proof. Represent the computation of Algorithm 1 as a tree of the recursion calls of enumerateMaximalChain, each node of which stores the current graph on which the recursion is called at line 11 . Of course, the root stores $G$ and on each leaf the condition Candidates $=\emptyset$ is true and a new solution is output. Observe that each leaf contains a feasible solution, and that no repetitions occur in view of Proposition 2, so the number of leaves is exactly $|C(G)|$.

Since at each call the size of $U$ is reduced by one, the tree height is necessarily bounded by $|U|=O(n)$; moreover, on each tree node, $O(\mathrm{~nm})$ time is spent for running function ComputeCandidates.

It follows that, since the algorithm explores the tree in DFS fashion starting from the root, between two solutions the running time is at most $O\left(n^{2} m\right)$ and the total running time is $O\left(|C(G)| n^{2} m\right)$.

\section{Upper bounds on the number of Maximal Chain Subgraphs}

In this section, we give two upper bounds on the maximum number of maximal chain subgraphs of a bipartite graph $G$ with $n$ nodes and $m$ edges. The first bound is given in terms of $n$ while the second depends on $m$. These bounds are of independent interest, however we will use them in two directions. First, they will allow us to determine the (input-sensitive) complexity of Algorithm 1. Indeed, in Proposition 3, we proved that the total running time of Algorithm 1 is of the form $O(D(n) \times|C(G)|)$, where $D(n)$ is the delay of the algorithm and $|C(G)|$ is the number of maximal chain subgraphs of $G$. Thus, a bound on $|C(G)|$ leads to a bound on the running time of Algorithm 1 depending on the size of the input. Second, the bound on $|C(G)|$ in terms of edges allows us to compute the time complexity of an exact exponential algorithm for the minimum chain subgraph cover problem in Section 5.

\subsection{Bound in terms of nodes}

The following lemma claims that a given permutation is the neighbourhood ordering of at most one maximal chain subgraph.

Lemma 1. Let $C_{1}$ and $C_{2}$ be two maximal chain subgraphs of $G=(U \cup W, E)$ and let $\pi_{1}$ (resp. $\pi_{2}$ ) be a neighbourhood ordering of $C_{1}$ (resp. $C_{2}$ ). Then, $\pi_{1}=\pi_{2} \Longrightarrow C_{1}=C_{2}$.

Proof. The proof proceeds by induction on the number of nodes of $U$.

If $|U|=1$ then $G$ has only one maximal chain subgraph and the result trivially holds.

Assume now that $|U|>1$. By Proposition 1, we have that $N_{C_{1}}\left(u_{\pi(|U|)}\right)=N_{G}\left(u_{\pi(|U|)}\right)=$ $N_{C_{2}}\left(u_{\pi(|U|)}\right)$. Using again Proposition 1, we obtain that $C_{1}^{\prime}:=C_{1}\left[U \backslash\left\{u_{\pi(|U|)}\right\}, N_{G}\left(u_{\pi(|U|)}\right)\right]$ and $C_{2}^{\prime}:=C_{2}\left[U \backslash\left\{u_{\pi(|U|)}\right\}, N_{G}\left(u_{\pi(|U|)}\right)\right]$ are maximal chain subgraphs of the graph defined as $G\left[U \backslash\left\{u_{\pi(|U|)}\right\}, N_{G}\left(u_{\pi(|U|)}\right)\right]$. Applying the inductive hypothesis with the permutations restricted to the $|U|-1$ elements, we have that $C_{1}^{\prime}=C_{2}^{\prime}$. Finally, since $N_{C_{1}}\left(u_{\pi(|U|)}\right)=$ $N_{C_{2}}\left(u_{\pi(|U|)}\right)$, we conclude that $C_{1}=C_{2}$. 
As a corollary, the maximum number of chain subgraphs of a graph $G=(U \cup W, E)$ is bounded by $|U|$ !. Since the same reasoning can be applied on $W$, we have that $|C(G)| \leq$ $|W|$ ! and hence:

$$
|C(G)| \leq \min (|U|,|W|) ! \leq \frac{n}{2} !
$$

This bound is tight as shown by the following family of graphs that reaches it.

Consider the antimatching graph with $n$ nodes $A_{n}=(U \cup W, E)$ defined as the complement of an $n / 2$ edge perfect matching, i.e.:

$$
\begin{aligned}
& U:=\left\{u_{1}, \ldots, u_{n / 2}\right\}, \quad W:=\left\{w_{1}, \ldots, w_{n / 2}\right\}, \\
& E:=\left\{\left(u_{i}, w_{j}\right) \in U \times W: i \neq j\right\}
\end{aligned}
$$

It is not difficult to convince oneself that the maximal chain subgraphs of $A_{n}$ are exactly $(n / 2)$ ! and that a different permutation corresponds to each of them. In particular, for each permutation $\pi$ of the nodes of $U$, the corresponding maximal chain subgraph $C_{\pi}$ of $A_{n}$ can be defined by means of the set of neighbourhoods as follows:

$$
N_{C_{\pi}}\left(u_{i}\right):=\left\{w_{k} \text { s.t. } \pi^{-1}(k)<\pi^{-1}(i)\right\} .
$$

The so-defined graph $C_{\pi}$ is a chain subgraph since all the neighbourhoods form a chain of inclusions. Moreover, it is maximal since if we added to the neighbourhood of $u_{i}$ any one of the missing edges $\left(u_{i}, w_{j}\right)$ with $\pi^{-1}(j) \geq \pi^{-1}(i)$, we would introduce a $2 K_{2}$ with the existing edge $\left(u_{j}, w_{i}\right)$ as $\left(u_{j}, w_{j}\right)$ and $\left(u_{i}, w_{i}\right)$ are not in $E$.

\subsection{Bound in terms of edges}

Let $T(m)$ be the maximum number of maximal chain subgraphs over all bipartite graphs with $m$ edges. We prove that $T(m) \leq 2^{\sqrt{m} \log (m)}$.

Lemma 2. Let $G=(U \cup W, E)$ be a bipartite graph. Then $|C(G)| \leq|U| \cdot T(m-|W|)$.

Proof. In view of how the algorithm works and of Proposition 1, at the beginning, there at most $|U|$ candidates. For each candidate $x$, we can build as many chain subgraphs as there are in $G\left[U \backslash\{x\}, N_{G}(x)\right]$. We claim that this latter graph has at most $m-|W|$ edges. Indeed, in order to construct $G\left[U \backslash\{x\}, N_{G}(x)\right]$, we remove from $G$ exactly $\left|E_{G}(x)\right|$ edges when deleting $x$ from $U$, and $|W|-\left|N_{G}(x)\right|$ nodes (each one connected to at least a different edge as $G$ is connected) when reducing $W$ to $N_{G}(x)$. Observing that $\left|E_{G}(x)\right|=$ $\left|N_{G}(x)\right|$, in total we remove $|W|$ edges. The proof follows from the fact that the number of chain subgraphs of $G\left[U \backslash\{x\}, N_{G}(x)\right]$ is bounded by $T(m-|W|)$.

Theorem 1. Let $G=(U \cup W, E)$ be a bipartite graph with $n$ nodes and $m$ edges; then $|C(G)| \leq 2^{\sqrt{m} \log m}$, i.e. $T(m) \leq 2^{\sqrt{m} \log m}$.

Proof. Assume w.l.o.g that $|U| \leq|W|$. The proof is by induction on $m$. Note that for $m=1$ the theorem holds trivially.

Applying the inductive hypothesis and Lemma 2, we have:

$$
|C(G)| \leq|U| T(m-|W|) \leq \frac{n}{2} 2\left(\sqrt{m-\frac{1}{2} n} \log \left(m-\frac{1}{2} n\right)\right) .
$$




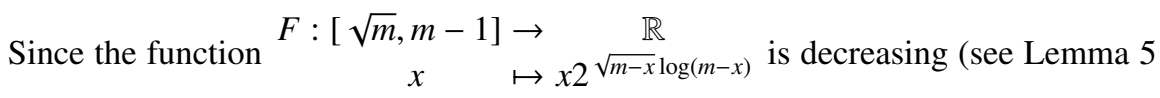
in Appendix), the maximum of $\frac{n}{2} 2 \sqrt{m-\frac{n}{2}} \log \left(m-\frac{n}{2}\right)$ is reached when $n / 2$ is minimum. Note that trivially for a bipartite graph we have $n / 2>\sqrt{m}$. Hence,

$$
|C(G)| \leq \sqrt{m} 2^{\sqrt{m-\sqrt{m}}} \log (m-\sqrt{m})
$$

Let $A:=\sqrt{m}-\sqrt{m-\sqrt{m}}$ and $B:=\frac{m-\sqrt{m}}{m}$. We then have:

$$
\begin{aligned}
|C(G)| & \leq \sqrt{m} 2^{(\sqrt{m}-A) \log (m B)} \\
& =2^{\sqrt{m} \log m} \times \sqrt{m} 2^{\log B(\sqrt{m}-A)-A \log (m)}
\end{aligned}
$$

Let us show that $Z:=\sqrt{m} 2^{\log B(\sqrt{m}-A)-A \log m} \leq 1$ by showing that $\log Z \leq 0$ :

$$
\begin{aligned}
\log Z & =\log \sqrt{m}+\log B(\sqrt{m}-A)-A \log (m) \\
& =\log \sqrt{m}(1-2 A)+\log B(\sqrt{m}-A) \\
& \leq 0
\end{aligned}
$$

considering that $B<1$ and $1 / 2<A \leq 1$ since:

$$
A=\frac{1}{1+\sqrt{B}}=\frac{1}{1+\sqrt{1-\frac{1}{\sqrt{m}}}}
$$

Corollary 1. The (input-sensitive) complexity of Algorithm 1 is bounded by $O^{*}\left(2^{\sqrt{m} \log (m)}\right)$.

\section{Minimum Chain Subgraph Cover}

In this section, we show how to find in polynomial space a minimum chain subgraph cover in time $O^{*}\left((2+\epsilon)^{m}\right)$, for every $\varepsilon>0$. Since a chain subgraph cover is a family of subsets of edges, the existence of an algorithm whose complexity is close to $2^{m}$ is not obvious. Indeed the basic search space has size $2^{2^{m}}$, as it corresponds to a family of subsets of edges. To obtain this result, we exploit Algorithm 1, the bound obtained in Theorem 1 and the inclusion/exclusion method $[1,8]$ that has already been successfully applied to exact exponential algorithms for many partitioning and covering problems.

We first express the problem as an inclusion-exclusion formula over the subsets of edges of $G$.

Proposition 4. [1] Let $c_{k}(G)$ be the number of chain subgraph covers of size $k$ of a graph $G$. We have that:

$$
c_{k}(G)=\sum_{A \subseteq E}(-1)^{|A|} a(A)^{k}
$$

where $a(A)$ denotes the number of maximal chain subgraphs not intersecting $A$.

Exploring this result brings to the exact algorithm as described in the proof of the next theorem. 
Theorem 2. Given a bipartite graph $G$ with $m$ edges, for all $k \in \mathbb{N}^{*}$ and for all $\varepsilon>0$, $c_{k}(G)$ can be computed in time $O^{*}\left((2+\epsilon)^{m}\right)$.

Proof. Let $G=(U \cup W, E)$ be a bipartite graph, $k \in \mathbb{N}^{*}$ and $\varepsilon>0$. Using the formula of Proposition $4, c_{k}$ can be computed in time $\sum_{i=0}^{m}\left(\begin{array}{c}m \\ i\end{array}\right) C(i)$, where $C(i)$ is the time complexity needed to compute $a(A),|A|=i$.

Notice that to compute $a(A)$ for a given $A \subseteq E$, one can naively compute all maximal chain subgraphs of $G^{\prime}=(U \cup W, E \backslash A)$ and, for each of them, check whether it is maximal in $G$. Using this fact, and Corollary $1, C(i)$ can be determined in time $O\left(n^{2} m 2^{\sqrt{m-i} \log (m-i)}\right)$.

Thus we have that $c_{k}(G)$ can be computed in time $\sum_{i=0}^{m}\left(\begin{array}{c}m \\ i\end{array}\right) n^{2} m 2^{\sqrt{m-i} \log (m-i)}$. Observe now that since $2^{\sqrt{m-i} \log (m-i)}=o\left((1+\varepsilon)^{m}\right)$, there exists a constant $n_{\varepsilon}$ such that for all $m>n_{\varepsilon}, 2^{\sqrt{m-i} \log (m-i)}<(1+\varepsilon)^{m}$.

Recalling that $G$ is connected and thus $m \geq n$, we then have:

$$
\begin{aligned}
\sum_{i=0}^{m}\left(\begin{array}{c}
m \\
i
\end{array}\right) n^{2} m 2^{\sqrt{m-i} \log (m-i)}= & n^{2} m\left(\sum_{i=0}^{m-n_{\varepsilon}-1}\left(\begin{array}{c}
m \\
i
\end{array}\right) 2^{\sqrt{m-i} \log (m-i)}+\sum_{i=m-n_{\varepsilon}}^{m}\left(\begin{array}{c}
m \\
i
\end{array}\right) 2^{\sqrt{m-i} \log (m-i)}\right) \\
& \leq n^{2} m\left(\sum_{i=0}^{m-n_{\varepsilon}-1}\left(\begin{array}{c}
m \\
i
\end{array}\right)(1+\varepsilon)^{m-i}+n_{\varepsilon} m^{n_{\varepsilon}} 2^{\sqrt{n_{\varepsilon}} \log \left(n_{\varepsilon}\right)}\right) \\
& \leq n^{2} m\left(\sum_{i=0}^{m}\left(\begin{array}{c}
m \\
i
\end{array}\right)(1+\varepsilon)^{m-i}+n_{\varepsilon} m^{n_{\varepsilon}} 2^{\sqrt{n_{\varepsilon}} \log \left(n_{\varepsilon}\right)}\right) \\
& \leq n^{2} m(2+\varepsilon)^{m}+n^{2} n_{\varepsilon} m^{1+n_{\varepsilon}} 2^{\sqrt{n_{\varepsilon}} \log \left(n_{\varepsilon}\right)} \\
& =O^{*}\left((2+\varepsilon)^{m}\right) .
\end{aligned}
$$

We conclude, by observing that the size of a minimum chain cover is given by the smallest value of $k$ for which $c_{k}(G) \neq 0$.

\section{Enumeration of Minimal Chain Subgraph Covers}

In this section, we prove that the enumeration of all minimal chain subgraph covers can be polynomially reduced to the enumeration of the minimal set covers of a hypergraph. This reduction implies that there is a quasi-polynomial time algorithm to enumerate all minimal chain subgraph covers. Indeed, the result in [9] implies that all the minimal set covers of a hypergraph can be enumerated in time $N^{\log N}$ where $N$ is the sum of the input size (i.e. $n+m)$ and of the output size (i.e. the number of minimal set covers).

Let $G=(U \cup W, E)$ be a bipartite graph, $C=C(G)$ the set of all its maximal chain subgraphs, and $\mathcal{S}=\mathcal{S}(G)$ the set of its minimal chain subgraph covers. Notice that the minimal chain subgraph covers of $G$ are the minimal set covers of the hypergraph $\mathcal{H}:=(V, \mathcal{E})$ where $V=E$ and $\mathcal{E}=C$. Unfortunately, the size of $\mathcal{H}$ might be exponential in the size of $G$ plus the size of $\mathcal{S}$. Indeed not every maximal chain subgraph in $C$ will necessarily be part of some minimal chain subgraph cover. In order to obtain a quasipolynomial time algorithm to enumerate all minimal chain subgraph covers, we need to 
enumerate only those maximal chain subgraphs that belong to a minimal chain subgraph cover.

Given an edge $e \in E$, let $C_{e}$ be the set of all maximal chain subgraphs of $G$ containing $e$ and $\mathcal{M}_{e}$ the set of all edges $e^{\prime} \in E$ inducing a $2 K_{2}$ in $G$ together with $e$.

We call an edge $e \in E$ non-essential if there exists another edge $e^{\prime} \in E$ such that $C_{e^{\prime}} \subset C_{e}$. An edge which is not non-essential is said to be essential. Note that for every non-essential edge $e$, there exists an essential edge $e_{1}$ such that $C_{e_{1}} \subset C_{e}$. Indeed, by applying iteratively the definition of a non-essential edge, we obtain a list of inclusions $C_{e} \supset C_{e_{1}} \supset C_{e_{2}} \ldots$, where no $C_{e_{i}}$ is repeated as the inclusions are strict. The last element of the list will correspond to an essential edge.

The following lemma claims that if a maximal chain subgraph $C$ contains at least one essential edge, then it belongs to at least one minimal chain subgraph cover.

Lemma 3. Let $C$ be a maximal chain subgraph of a bipartite graph $G=(U \cup W, E)$. Then $C$ belongs to a minimal chain subgraph cover of $G$ if and only if $C$ contains an essential edge.

Proof. $(\Rightarrow)$ Let $C$ belong to a minimal chain subgraph cover $M$ and assume that $C$ contains no essential edge. Given $e \in C$, $e$ therefore being non-essential, there exists an essential edge $e^{\prime}$ such that $C_{e^{\prime}} \subset C_{e}$. Moreover, $e^{\prime} \notin C$. As $M$ is a cover, there exists $C^{\prime} \in M$ such that $e^{\prime} \in C^{\prime}$. Thus, $C^{\prime} \neq C, C^{\prime} \in C_{e^{\prime}} \subset C_{e}$, hence $e \in C^{\prime}$. Since for every edge $e \in C$, there exists $C^{\prime} \in M$ containing it, we have that $M \backslash\{C\}$ is a cover, contradicting the minimality of $M$.

$(\Leftarrow)$ Assume $C$ contains an essential edge $e$. Let $C^{\prime}=\{D \in C(G): e \notin D\}$. Note that $C^{\prime}=C \backslash C_{e}$. We show that $C^{\prime} \cup\{C\}$ is a cover. Suppose on the contrary that there exists $e^{\prime} \in E \backslash E(C)$ and $e^{\prime}$ is not covered by $C^{\prime}$ and thus $C_{e^{\prime}} \cap C^{\prime}=\emptyset$. This implies that $C_{e^{\prime}} \subseteq C \backslash C^{\prime}=C_{e}$ and as $e$ is essential, we obtain $C_{e^{\prime}}=C_{e}$ from which we deduce that $e^{\prime} \in C$. Thus, $M=C^{\prime} \cup\{C\}$ is a cover and clearly it contains a minimal one. Finally, we conclude by observing that, since by construction $C$ is the only chain subgraph of $M$ that contains $e$, it belongs to any minimal cover contained in $M$.

It follows that the set of maximal chain subgraphs that can contribute to a minimal chain cover is $\tilde{C}=\cup C_{e}$ where the index $e$ of the union operation runs over all the essential edges of $G$. In the following, we show how to detect essential edges. This problem then consists in detecting all the couples $e_{1}, e_{2}$ such that $C_{e_{1}} \subseteq C_{e_{2}}$ before enumerating all useful maximal chain subgraphs. The next lemma is proved in Appendix.

Lemma 4. Let $C$ be a maximal chain subgraph of a bipartite graph $G=(U \cup W, E)$ and let $e \in E$ be such that for all $e^{\prime} \in E(C)$, it holds that $e \notin \mathcal{M}_{e^{\prime}}$. Then $e \in C$.

Using this lemma we can now prove the following result.

Theorem 3. Given a bipartite graph $G=(U \cup W, E)$, for any two edges e, $e^{\prime} \in E$, $C_{e} \subseteq C_{e^{\prime}}$ if and only if $\mathcal{M}_{e} \supseteq \mathcal{M}_{e^{\prime}}$.

Proof. $(\Rightarrow)$ Given two edges $e, e^{\prime} \in E$, suppose that $C_{e} \subseteq C_{e^{\prime}}$, and assume on the contrary that there exists $f \in \mathcal{M}_{e^{\prime}}$ and $f \notin \mathcal{M}_{e}$. Then there exists a maximal chain 
$C^{\prime}$ containing $e$ and $f$ (as they do not form a $2 K_{2}$ in $G$ ) but not $e^{\prime}\left(f \in \mathcal{M}_{e^{\prime}}\right)$. Hence, $C^{\prime} \in C_{e}$ but $C^{\prime} \notin C_{e^{\prime}}$, contradicting the assumption that $C_{e} \subseteq C_{e^{\prime}}$.

$(\Leftarrow)$ Suppose now $\mathcal{M}_{e} \supseteq \mathcal{M}_{e^{\prime}}$. Let $C \in C_{e}$. By definition, none of the edges of $\mathcal{M}_{e}$ appears in $C$. Hence, $e^{\prime}$ does not form a $2 K_{2}$ with any edge in $C$ in the graph $G$ (as $\left.\mathcal{M}_{e} \supseteq \mathcal{M}_{e^{\prime}}\right)$. By Lemma $4 e^{\prime} \in C$. Thus, $C_{e} \subseteq C_{e^{\prime}}$.

Notice that, given an edge $e=(u, w) \in E, u \in U$ and $w \in W$, it is easy to determine the set $\mathcal{M}_{e}$. We just need to start from $E$ and delete all edges that are incident either to $u$ or to $w$, as well as all edges at distance 2 from $e$ (that is all edges $e^{\prime}=\left(u^{\prime}, w^{\prime}\right)$ such that either $u^{\prime}$ is adjacent to $w$ or $w^{\prime}$ is adjacent to $u$ ). Checking whether $\mathcal{M}_{e} \supseteq \mathcal{M}_{e^{\prime}}$ is also easy: it suffices to sort the edges in each set in lexicographic order, and then the inclusion of each pair can be checked in linear time in their size, that is in $O(m)$. It is thus possible to enumerate in polynomial delay only those maximal chain subgraphs that contain at least one essential edge by modifying Algorithm 1. Due to space limits, we do not detail the algorithm here and refer instead the reader to Algorithm 2 in the Appendix. Finally, we are now able to state the main result of this section.

Theorem 4. Given a bipartite graph $G=(U \cup W, E)$, one can enumerate all its minimal chain subgraph covers, i.e. all the elements in $\mathcal{S}$, in time $O\left(|\mathcal{S}|^{\log (|\mathcal{S}|)+2}\right)$.

Proof. We first construct the hypergraph $\mathcal{H}=(V, \mathcal{E})$ where $V:=E^{\prime}$ is the set of essential edges of $G$ and $\mathcal{E}:=C_{e s s}$ is the set of maximal chain subgraphs of $G$ that contain at least one essential edge. This takes time $O\left(n^{2} m \mid C_{e s s}\right)$. Applying then the algorithm given in [9], one can enumerate all minimal set covers of $\mathcal{H}$ (i.e. all minimal chain subgraph covers $)$ in time $O\left((|\mathcal{H}|+|\mathcal{S}|)^{\log (|\mathcal{H}|+|\mathcal{S}|)}\right)=O\left(\left(\left|C_{\text {ess }}\right|+|\mathcal{S}|\right)^{\log \left(||_{\text {ess }}|+| \mathcal{S} \mid\right)}\right)$. The total running time is thus $O\left(n^{2} m\left|C_{e s s}\right|+\left(\left|C_{e s s}\right|+|\mathcal{S}|\right)^{\log \left(\left|C_{e s s}\right|+|\mathcal{S}|\right)}\right)$. Notice now that since by Lemma 3, every maximal chain subgraph in $C_{\text {ess }}$ belongs to at least one minimal chain subgraph cover, we have that $\left|C_{e s s}\right| \leq|\mathcal{S}|$. Finally, we obtain that the total running time is $O\left(n^{2} m|\mathcal{S}|+(|\mathcal{S}|+|\mathcal{S}|)^{\log (|\mathcal{S}|+|\mathcal{S}|)}\right)=O\left(|\mathcal{S}|^{\log (|\mathcal{S}|)+2}\right)$.

\section{Conclusion}

In this paper, we studied different problems related to maximal chain subgraphs and chain subgraph covers in bipartite graphs. This work raises many questions. First, it remains an open problem whether it is possible to enumerate the minimal chain covers of a graph in polynomial delay. Indeed, our problem is more constrained than an arbitrary instance of the set cover of a hypergraph. A future goal is to better exploit the connections between these two problems. Second, it would be interesting to determine the exact value of $T(m)$. We conjecture that a tighter bound may be $2^{\frac{1+\sqrt{1+4 m}}{2}}$. Finally, it is worth exploring the different nature of the problems considered here in the case where we deal with an hereditary property (induced chain subgraphs) instead of a non-hereditary one (non necessarily induced chain subgraphs). In particular, it remains unknown whether enumerating maximal induced subgraphs can be done in polynomial delay. 


\section{Acknowledgments}

T. Calamoneri is supported in part by the Italian Ministry of Education, University, and Research (MIUR) under PRIN 2012C4E3KT national research project "AMANDA - Algorithmics for MAssive and Networked DAta" and in part by Sapienza University of Rome project "Graph Algorithms for Phylogenetics: A Promising Approach". M. Gastaldello is supported by the Università Italo-Francese project "Algorithms and Models for the solution of difficult problems in biology". A. Mary is supported by the ANR project GraphEN "Enumération dans les graphes et les hypergraphes: algorithmes et complexité", ANR-15-CE40-0009.

\section{References}

1. Andreas Björklund, Thore Husfeldt, and Mikko Koivisto. Set partitioning via inclusionexclusion. SIAM J. Comput., 39(2):546-563, July 2009.

2. Béla Bollobás. Modern graph theory, volume 184 of Graduate Texts in Mathematics. Springer-Verlag, Berlin, 1998.

3. A. Brandstädt, E. M Eschen, and R. Sritharan. The induced matching and chain subgraph cover problems for convex bipartite graphs. Theoretical computer science, 381(1):260-265, 2007.

4. Yu Chang-Wu, Chen Gen-Huey, and Ma Tze-Heng. On the complexity of the k-chain subgraph cover problem. Theoretical computer science, 205(1):85-98, 1998.

5. Vânia M.F. Dias, Celina M.H. de Figueiredo, and Jayme L. Szwarcfiter. Generating bicliques of a graph in lexicographic order. Theoretical Computer Science, 337(1-3):240 - 248, 2005.

6. Vânia M.F. Dias, Celina M.H. de Figueiredo, and Jayme L. Szwarcfiter. On the generation of bicliques of a graph. Discrete Applied Mathematics, 155(14):1826 - 1832, 2007.

7. Thomas Eiter and Georg Gottlob. Identifying the minimal transversals of a hypergraph and related problems. SIAM Journal on Computing, 24(6):1278-1304, 1995.

8. Fedor V. Fomin and Dieter Kratsch. Exact Exponential Algorithms. Springer-Verlag New York, Inc., New York, NY, USA, 2010.

9. M. L Fredman and L. Khachiyan. On the complexity of dualization of monotone disjunctive normal forms. Journal of Algorithms, 21(3):618-628, 1996.

10. D. S. Johnson, M. Yannakakis, and C. H. Papadimitriou. On generating all maximal independent sets. Information Processing Letters, 27(3):119-123, 1988.

11. Kazuhisa Makino and Takeaki Uno. SWAT 2004, Lecture Notes in Computer Science, chapter New Algorithms for Enumerating All Maximal Cliques, pages 260-272. Springer Berlin Heidelberg, Berlin, Heidelberg, 2004.

12. J. W. Moon and L. Moser. On cliques in graphs. Israel Journal of Mathematics, 3(1):23-28, 1965.

13. I. Nor, J. Engelstädter, O. Duron, M. Reuter, M-F. Sagot, and S. Charlat. On the genetic architecture of cytoplasmic incompatibility: inference from phenotypic data. The American Naturalist, 182(1):E15-E24, 2013.

14. Mihalis Yannakakis. The complexity of the partial order dimension problem. SIAM Journal on Algebraic Discrete Methods, 3(3):351-358, 1982. 


\section{Appendix}

\section{Upper bounds on the number of Maximal Chain Subgraphs}

We provide here the proof of the following result that was used in the proof of Theorem 1 .

Lemma 5. The function:

$$
\begin{aligned}
F:[\sqrt{m}, m-1] & \rightarrow \quad \mathbb{R} \\
x & \mapsto x 2^{\sqrt{m-x} \log (m-x)} \\
\text { is decreasing. } &
\end{aligned}
$$

Proof. The derivative of $F(x)$ is given by:

$$
\begin{aligned}
& -x\left(\frac{\log (m-x)}{2 \sqrt{m-x}}+\frac{1}{\sqrt{m-x}}\right) 2^{(\sqrt{m-x} \log (m-x))}+2^{(\sqrt{m-x} \log (m-x))} \\
= & -\frac{(x \log (m-x)+2 x-2 \sqrt{m-x}) 2^{(\sqrt{m-x} \log (m-x))}}{2 \sqrt{m-x}}
\end{aligned}
$$

Then the derivative is negative whenever $(x \log (m-x)+2 x-2 \sqrt{m-x}) \geq 0$.

Observe that $\log (m-x) \geq 0$ for $x \leq m-1$, while for $x \geq 0$ we have:

$$
2 x-2 \sqrt{m-x} \geq 0 \Longleftrightarrow x \geq \frac{-1+\sqrt{1+4 m}}{2}=-\frac{1}{2}+\sqrt{m+\frac{1}{4}}
$$

and:

$$
\sqrt{m} \geq-\frac{1}{2}+\sqrt{m+\frac{1}{4}}
$$

\section{Enumeration of Minimal Chain Subgraph Covers}

We prove here Lemma 4. We start by showing the following fact.

Fact 1 Let $C=(X \cup Y, F)$ be a maximal chain subgraph of a bipartite graph $G=$ $(U \cup W, E)$, and let $z \in X, e=\{u, w\} \in E$ be such that for every $e^{\prime} \in E_{C}(z)$, we have $e \notin \mathcal{M}_{e^{\prime}}$. Then at least one of the following holds:

(1) $w \in N_{G}(z)$.

(2) $u \in \cap_{y \in N_{C}(z)} N_{G}(y)$.

Proof. The proof follows straightforwardly by observing that for any $e^{\prime}=\{z, y\} \in C$ then as $e \notin \mathcal{M}_{e^{\prime}}$, either $\{z, w\} \in E(G)$ or $\{u, y\} \in E(G)$.

Lemma 4. Let $C$ be a maximal chain subgraph of a bipartite graph $G=(U \cup W, E)$ and let $e \in E$ be such that for all $e^{\prime} \in E(C)$, it holds that $e \notin \mathcal{M}_{e^{\prime}}$. Then $e \in C$. 
Proof. Let $C=(X \cup Y, F)$ be a maximal chain subgraph of $G=(U \cup W, E)$ and w.l.o.g., let $N_{C}\left(u_{1}\right) \subseteq N_{C}\left(u_{2}\right) \subseteq \ldots \subseteq N_{C}\left(u_{|X|}\right)$. Let $e=\{u, w\}$ in $E$ be such that for all $e^{\prime} \in E(C)$, it holds that $e \notin \mathcal{M}_{e^{\prime}}$. Assume that $e \notin C$.

We will show that $w \in \cap_{x \in X} N_{G}(x)$ leads to a contradiction because we could add all the edges $E_{G}(w)$ to $C$ and still obtain a chain subgraph (with $N_{G}(w)$ as the largest neighbourhood of $C$ ).

Observe that in the previous claim, we can re-write (2) in the form $N_{C}(z) \subseteq N_{G}(u)$.

Using the previous fact with $z=u_{|X|}$, we have that at least one from (1) and (2) must hold. Observe that (2) cannot hold as otherwise it implies that $u \in \cap_{y \in N_{C}(z)} N_{G}(y)$. We are then done by interchanging the roles of $Y$ and $X$ and observing by point (i) of Proposition 1 that $N_{C}\left(u_{|X|}\right)=N_{G}\left(u_{|X|}\right)=Y$. Thus, (1) must hold, i.e. $w \in N_{G}\left(u_{|X|}\right)$.

If we now show that $w \in \cap_{k=j}^{|X|} N_{G}\left(u_{k}\right) \Rightarrow w \in N_{G}\left(u_{j-1}\right)$, we are done since together with $w \in N_{G}\left(u_{|X|}\right)$ this leads to:

$$
w \in \bigcap_{k=1}^{|X|} N_{G}\left(u_{k}\right)=\bigcap_{x \in X} N_{G}(x)
$$

To prove this, we apply again Fact 1 with $z=u_{j-1}$ and show that (2) cannot hold. Notice that from (2), we have $N_{C}\left(u_{j-1}\right) \subseteq N_{G}(u)$. Then using the maximality of $C$, we deduce that $u \in X$. Indeed, $N_{C}(u)$ has to contain at least $N_{C}\left(u_{j-1}\right)$, and hence there exists $\tilde{k} \geq j-1$ for which $u=u_{\tilde{k}}$. Then we could extend $C$ to $C^{\prime}$ by adding $e$. Observe that $C^{\prime}$ has the following list of neighbourhoods:

$$
\begin{array}{ll}
N_{C^{\prime}}\left(u_{k}\right):=N_{C}\left(u_{k}\right) & \text { for } k \neq \tilde{k} \\
N_{C^{\prime}}\left(u_{k}\right):=N_{C}\left(u_{k}\right) \cup\{w\} & \text { for } k=\tilde{k}
\end{array}
$$

and is a chain subgraph since $N_{C}\left(u_{\tilde{k}}\right) \cup\{w\} \subseteq N_{C}\left(u_{k}\right)$ for all $k>\tilde{k} \geq j-1$ by $w \in$ $\cap_{k=j}^{|X|} N_{G}\left(u_{k}\right)$ and the maximality of $C$. 


\section{Description of Algorithm 2.}

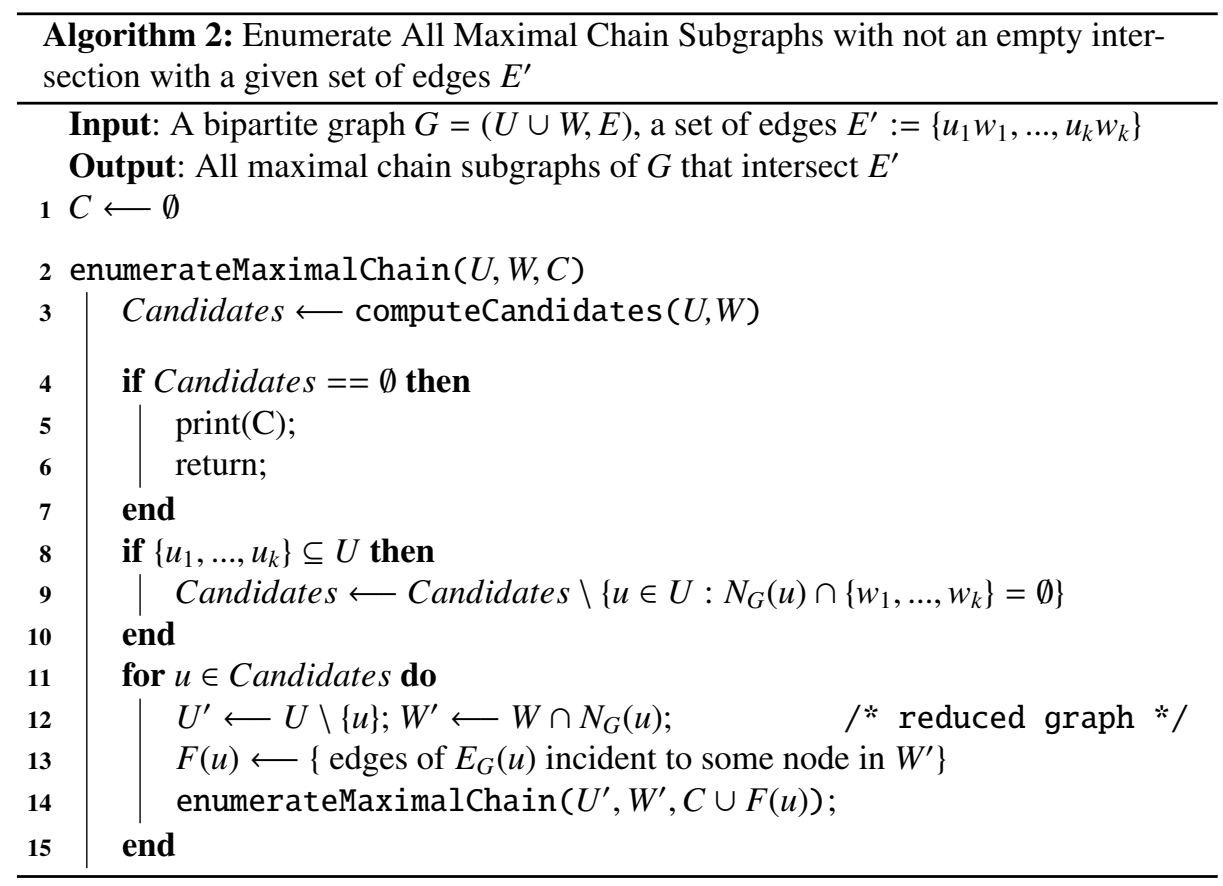

